\title{
Modeling the body temperature throughout the day with a two-term function
}

\author{
HELENA ALMIRALL \\ University of Barcelona, Barcelona, Spain
}

\begin{abstract}
A two-term function with potential and exponential terms is proposed to model the body temperature throughout the day. These terms can be related to thermogenic and thermolytic processes that are opposite but simultaneous. The first term, a potential function, reflects that the heat gain process is predominant; the second term is a negative exponential function and reflects the predominance of heat dissipation. The function also provides the time of the inflection between thermal opposite processes. The proposed two-term function and a cosine function were fitted to 335 temperature series of 24 -h duration recorded in humans and animals. The percentage of variance accounted for by the two-term function was higher than that for the cosine approximation for the total sample and for those series whose shape was asymmetrical $(n=75)$. Nevertheless, no significant differences between the percentage of variance accounted for by the two functions were found for those series whose shape was symmetrical $(n=260)$. The results of different subgroups that differed in experimental conditions are discussed.
\end{abstract}

Although the pattern of a biological circadian rhythm is rarely symmetrical, circadian rhythmicity has traditionally been assessed by a sine function or a cosine wave that assumes that the circadian rhythm adjusts to a cosine symmetrical wave of $24 \mathrm{~h}$. For example, in human body temperature, the time between the minimum and the maximum thermal values is approximately $16 \mathrm{~h}$, and the time between the maximum and the minimum is $8 \mathrm{~h}$. Likewise, the ascending part is longer than the declining part, and it takes more than $12 \mathrm{~h}$ to reach the maximum value of body temperature from the thermal minimum, which usually occurs during sleep.

Alternative proposals to the cosine function have appeared. Minors and Waterhouse (1988) modeled a circadian rhythm with a mixture of the fundamental period of a cosine curve and various harmonics. In their opinion, the circadian system can behave as an oscillator and the use of harmonics can be particularly valuable when ultradian rhythms are being investigated, if summed to the circadian one. More drastically, Refinetti (1992) did not assume a cosine shape in the circadian rhythm of body temperature and, instead, studied the frequency histogram for each value of body temperature.

Although disagreement exists regarding how to model circadian rhythms, it is widely accepted that circadian rhythms have endogenous and exogenous components. The constant routine technique tries to minimize exogenous influences. In contrast, special conditions such as continuous light, suprachiasmatic lesions, and shift work maximize exogenous influences.

This work has been in part supported by Grants PB93-0752 from the Direccion General de Investigación Cientifica y Técnica from the government of Spain. Correspondence should be addressed to H. Almirall, Dept. of Psychiatry and Clinical Psychology, Univ. of Barcelona, Psg. de la Vall d'Hebron 171,08035 Barcelona, Spain (e-mail: ealmirall@psi.ub.es).
The approach presented in this study focuses on body temperature as the result of thermogenic and thermolytic processes that are opposite but simultaneously occurring at different paces during the day. Heat production is supplied by the metabolic rate, by voluntary activity of the locomotor apparatus, by tonic or rhythmic muscular activity, and by nonshivering thermogenesis in the newborn and in small, cold-adapted animals. Heat loss (thermolytic) processes can be divided into radiative, convective, evaporative, and conduction heat loss. Expressed in these terms, body temperature is one particular case of general biological phenomena caused by opposite forces, independently of their time scale.

The present study proposes that body temperature can be described by the two-term function used by Prodan (1968) to describe plant growth processes. This function consists of a potential term and an exponential term. The form of this function has an initial accelerated rise, resulting from the potential term (which can be interpreted as a constructive process), a plateau, and finally a decay resulting from the exponential term (which can be interpreted as a destructive process). This function also explains well the kinetics of a pharmacological response in which the concentration of a drug in blood is dependent on both absorption and elimination processes - that is, opponent processes partially delayed in time. As Warren (1980) pointed out, the function has great flexibility of form.

The function proposed to model daily body temperature is $y=a t^{b} e^{-c t} \varepsilon_{t}$, where $t$ is time, $a$ represents the departure point at some $t$ different from zero (the beginning of the measurements), $b$ the coefficient of the potential part of the function, $c$ the coefficient of the exponential part, and $\varepsilon_{t}$ an error factor. Moreover, the ratio $b / c$ informs of the time of the inflection point between the potential and exponential terms. The logarithmic transform of this 
model has the following form: $z=\alpha+\beta \ln t+\chi t+\delta_{t}$, where $z$ is $\ln y, \alpha$ is $\ln a, \beta$ is $b, \chi$ is $-c$, and $\delta_{t}$ is $\ln \varepsilon_{t}$.

The two-term function applied to the daily body temperature, choosing the thermal minimum as a departure point, would suppose a set of thermogenic mechanisms as dominant until reaching the thermal highest value. Thermolytic mechanisms would have an advantage from this point until the next thermal minimum. Obviously, many other influences should be superimposed onto this trend; for example, ultradian rhythms, food and activity effects, and so on. These influences would make the actual daily temperature curve "noisy."

The aim of this paper is to classify the shape of the body temperature throughout the day in humans and animals as symmetrical or asymmetrical and to apply the twoterm function to the evolution of this parameter. The sensitivity of the two-term function is tested and compared with a cosine function.

\section{METHOD}

A total of 60 articles from the circadian rhythm literature were reviewed, and 353 data graphs corresponding to circadian temperature cycles were selected. These data graphs could be classified according to the interval sample of the registers. In most cases, registers of circadian rhythms are obtained continuously or every hour, but in a few cases they are obtained every $90 \mathrm{~min}$ or $2 \mathrm{~h}$. To fit the two-term function with a margin of accuracy, 25 values constitute an acceptable number. Likewise, data graphs that were not obtained by continuous registers or hourly samples were discarded. For this reason, of the original sample of 353 data graphs, 18 (all of them from human studies) were disregarded. That means that in hourly sampled data graphs, the hourly temperature values were just read. In continuous records data graphs, only 25 equally spaced values were considered.

Of the total final sample of 335 graphs, 262 graphs (78.2\%) corresponded to human circadian temperature series and $73(21.8 \%)$ to animal series. The human series could be classified into five groups. Group 1 contained basal conditions, normative data, and entrained subjects $(17.2 \%)$. Group 2 contained constant routine studies $(3.8 \%)$. Group 3 dealt with free-running subjects (29.0\%). Group 4 assembled conditions, such as recovery from sleep deprivation, personality traits, pregnancy, depression, mild cold stress, and sleep disturbance, and constitutes a heterogeneous group (43.5\%). Group 5 was formed by shift-work studies $(6.5 \%)$. The animal sample could be classified in three groups. Group 1 contained basal conditions (i.e., 12:12-h light:dark schedule) and was the largest group $(64.4 \%)$. Group 2 involved suprachiasmatic nucleus lesions $(20.5 \%)$. Group 3 was formed with free-running animals (15.1\%).

To fit the two-term function requires that all the data be positive; for this reason, the thermal minimum was chosen as the first point, and an entire cycle was considered from a thermal minimum to the next thermal minimum. When the start of the registers did not coincide with the thermal minimum, as in most graphs, double-plot graphs were obtained.

The graphs of the literature can also be classified into three types according to the length of the data display; in the first, a single cycle is shown in a single plot; in the second, a double plot of a single cycle is presented; and the third shows successive cycles of the same subject. In the first case, double plots of the graphs were obtained by photocopies. In the third case, only complete cycles running from one thermal minimum to the next thermal minimum were considered.
Finally the data graphs studied could also vary in terms of the temporal scale depicted - actual or relative time. In the present study, the relative time scale was adopted, as is appropriate for testing the proposed model.

All the graphs were scanned and submitted to a graph program (Apple System), and 25 hourly temperature data values were obtained from all of them. The $x$-axis was normalized simply by picking 25 values along each graph. To homogenize the scale of the $y$-axis, the range between the maximum and the minimum for each series was taken as the unity.

SCA statistical software (Liu, Hudak, Box, Muller, \& Tiao, 1985 ) was used to calculate the skewness of each series and to fit the two-term and the cosine functions. For the two-term function, the calculations were made considering the lowest thermal value of each series as the baseline point. Thus all the values of the temperature series were positive and reflected the increase over the thermal minimum. The logarithmic transform of the two-term function was calculated, and the values of the parameters were estimated by the method of least squares. For the cosine function, a constant positive term was added to the sinusoidal function, and the phase position of it was varied in order to calculate the best fit.

\section{RESULTS}

Each series was classified as symmetrical or asymmetrical on the basis of whether its absolute skewness value was greater than .4637 , the fixed value of the standard error for a distribution of 25 data points. Of the whole sample, 75 series were asymmetrical and 260 were symmetrical. In the human sample, 51 series were asymmetrical and 211 symmetrical. In the animal sample, there were 24 asymmetrical series and 49 symmetrical. The proportion of symmetrical series in the human sample was greater than in the animal sample $\left[\chi^{2}(1)=\right.$ $5.16, p<.03]$.

The numbers of asymmetrical versus symmetrical series for each of the human groups were as follows: Group 1, 10 versus 35; Group 2, 3 versus 7; Group 3, 15 versus 61; Group 4, 19 versus 95; and Group 5, 4 versus 13. These different proportions were not statistically significant $\left[\chi^{2}(4)=1.68, p>.79\right]$. With respect to the animal groups, the numbers of asymmetrical versus symmetrical series were as follows: Group 1, 13 versus 34; Group 2, 7 versus 8; and Group 3, 4 versus 7. These proportions also were not statistically significant $\left[\chi^{2}(2)=\right.$ $1.93, p>.38]$.

Table 1 shows the means and standard errors of the percentage of variance accounted for by the two-term function and the cosine function for the human and animal samples and the groups analyzed. Figure 1 presents the body temperature throughout the day of a man in basal conditions, together with the two-term and cosine approximations.

The percentage of variance accounted for by the twoterm function did not statistically differ from the percentage accounted for by the cosine function, either in the whole human sample or in any of the five studied groups. On the contrary, in the whole animal sample the percentage of variance accounted for by the two-term function 
Table 1

Differences in the Variance Accounted for by Each Function According to the Human Sample or Animal Sample and the Groups

\begin{tabular}{|c|c|c|c|c|c|c|c|}
\hline & \multirow[b]{2}{*}{$n$} & \multicolumn{2}{|c|}{ Two-Term } & \multicolumn{2}{|c|}{ Cosine } & \multicolumn{2}{|c|}{$\begin{array}{c}\text { Wilcoxon Signed } \\
\text { Rank } \\
\end{array}$} \\
\hline & & $M$ & $S E M$ & $M$ & $S E M$ & $z$ & $p$ \\
\hline \multicolumn{8}{|l|}{ Human Sample } \\
\hline Basal & 45 & .78 & .02 & .76 & .02 & -0.17 & .86 \\
\hline Constant routine & 10 & .77 & .04 & .69 & .07 & -0.05 & .96 \\
\hline Free running & 76 & .73 & .02 & .68 & .03 & -1.23 & .22 \\
\hline Various & 114 & .66 & .02 & .65 & .02 & -0.08 & .94 \\
\hline Shift work & 17 & .67 & .04 & .61 & .07 & -1.02 & .31 \\
\hline Total & 262 & .70 & .01 & .67 & .01 & -0.96 & .33 \\
\hline \multicolumn{8}{|l|}{ Animal Sample } \\
\hline Basal & 47 & .69 & .03 & .47 & .03 & -4.76 & .0001 \\
\hline Lesioned & 15 & .68 & .05 & .61 & .03 & -1.14 & .26 \\
\hline Free running & 11 & .60 & .09 & .46 & .11 & -2.13 & .03 \\
\hline Total & 73 & .67 & .02 & .49 & .03 & -5.29 & .0001 \\
\hline Total Sample & 335 & .70 & .01 & .64 & .01 & -3.71 & .0002 \\
\hline
\end{tabular}

was greater than that accounted for by the cosine function. When the different animal groups were analyzed separately, the results appeared to be due to the basal and freerunning conditions, because in lesioned animals statistical significance was not reached.

The Wilcoxon signed-rank test for large samples was used to compare the sensitivity of the two models. If the asymmetric and symmetric series are considered separately, the two-term function fit better than the cosine function for the asymmetrical series in the human sample $(z=2.65, p<.008)$, in the animal sample $(z=3.945$, $p<.0001)$, and in the total sample $(z=4.73, p<.0001)$. In the symmetrical series, the two-term function fit better only in the animal sample $(z=3.61, p<.003)$.
With respect to the different groups, comparisons between the asymmetric versus symmetric series were only made if the number of series was equal or higher than 10that is, in Groups 1 (basal), 3 (free running), and 4 (various conditions) of the human sample and in Group 1 (basal) of the animal sample. These comparisons are shown in Table 2 . In asymmetrical series of the freerunning human group, the two-term function fit better than the cosine function, whereas in the animal basal group, the two-term function fit better than the cosine function in both the asymmetrical and in the symmetrical series.

The index $b / c$ of the two-term function was $10.37 \pm$ $.29,9.91 \pm .81,10.40 \pm .28,9.30 \pm .19$, and $9.93 \pm .25$

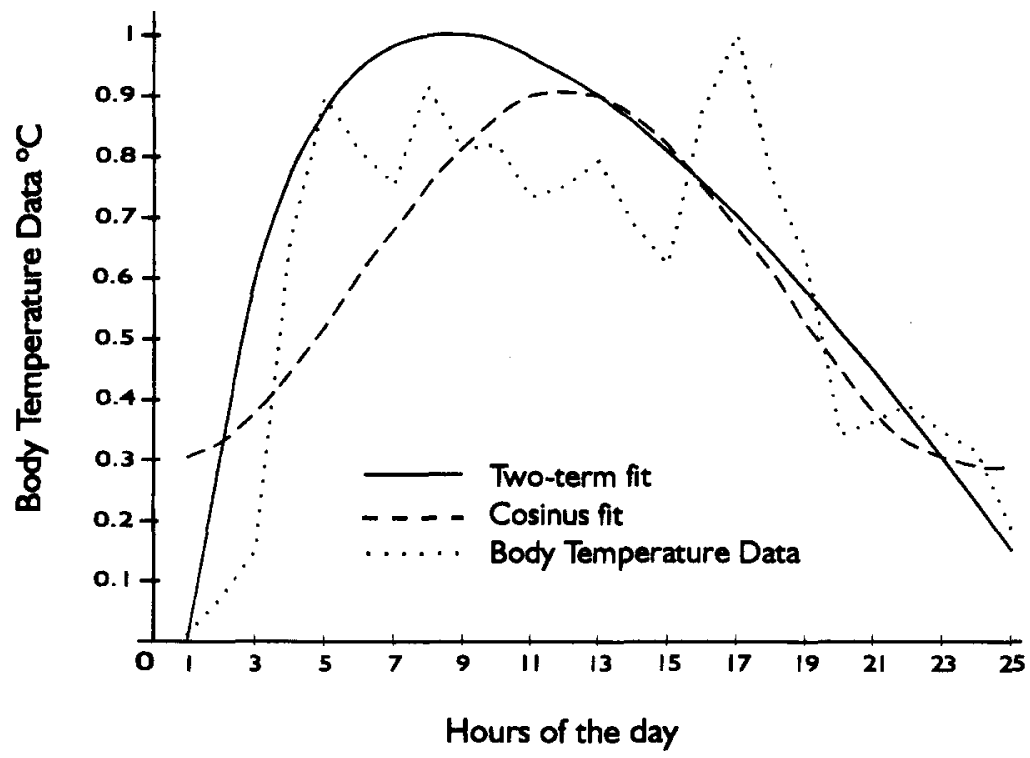

Figure 1. Plot showing the temperature series together with the two-term and cosine approximation. The ordinate values represent the differences between each actual temperature value and the minimum of temperature. 
Table 2

Differences in the Variance Accounted for by Each Function According to the Skewness of the Series

\begin{tabular}{|c|c|c|c|c|c|c|c|c|c|c|c|c|}
\hline & \multicolumn{6}{|c|}{ Asymmetric } & \multicolumn{6}{|c|}{ Symmetric } \\
\hline & \multirow[b]{2}{*}{$n$} & \multicolumn{2}{|c|}{ Two-Term } & \multicolumn{2}{|c|}{ Cosine } & \multirow[b]{2}{*}{$p$} & \multirow[b]{2}{*}{$n$} & \multicolumn{2}{|c|}{ Two-Term } & \multicolumn{2}{|c|}{ Cosine } & \multirow[b]{2}{*}{$p$} \\
\hline & & $M$ & $S E M$ & $M$ & $S E M$ & & & $M$ & $S E M$ & $M$ & $S E M$ & \\
\hline \multicolumn{13}{|l|}{ Human Sample } \\
\hline Basal & 10 & .77 & .04 & .71 & .03 & .58 & 35 & .78 & .02 & .77 & .02 & .82 \\
\hline Free-running & 15 & .77 & .05 & .54 & .07 & .006 & 61 & .73 & .02 & .71 & .03 & .99 \\
\hline Various & 19 & .59 & .04 & .55 & .06 & .64 & 95 & .67 & .02 & .67 & .02 & .75 \\
\hline \multicolumn{13}{|l|}{ Animal Sample } \\
\hline Basal & 13 & .72 & .05 & .42 & .05 & .002 & 34 & .68 & .03 & .48 & .04 & .003 \\
\hline
\end{tabular}

for the basal, constant-routine, free-running, variousconditions, and shift-work human groups, respectively. The value of $H$ provided by a Kruskal-Wallis test was $15.91(d f=4, p<.004)$, and so there were differences between these groups. Mann-Whitney comparisons showed that Group 4 statistically differed from Group $1\left[U^{\prime}=\right.$ $3,340, p<.001]$ and Group $3\left[U^{\prime}=5,400, p<.002\right]$. The value of this index was $10.29 \pm .42,10.65 \pm .91$, and $11.62 \pm .98$ for the basal, lesioned, and free-running animal groups, respectively. These differences did not reach statistical significance.

The other parameters of the two functions are not relevant because the $y$-axis had been previously normalized, as noted.

\section{DISCUSSION}

First, although the ascending part of body temperature is longer than the declining part, only 75 series were asymmetrical, whereas 260 series were symmetrical. This was an unexpected result, probably due to the high value of the standard error, which is a fixed value that depends on the number of data points. In the present study, to homogenize the graphs, the number of data points chosen for each graph was constant and data were equally time spaced, although in some cases the temperature was obtained continuously, and more data points could have been obtained. In this case, the standard error would have been lower, and probably more series would have been classified as asymmetrical.

Second, the two-term function seems to be useful in modeling the body temperature rhythm. Nevertheless, the shape of the distribution appears to have an important role. Results show that when the series is asymmetrical, the two-term function accounts for a greater percentage of variance than does the cosine function. Asymmetrical series predominate in the animal sample when compared with the human sample. This could be due to (1) a specific human endogenous effect, (2) strong exogenous effects that prevail in human experiments, or (3) a less accurate control of human experiments compared with animal experiments. The percentage of variance accounted for by the two-term function was higher in the animal sample than the variance accounted for by the cosine function in both the basal and free-running conditions. There were no significant differences in the suprachiasmatic-lesion con- dition that could be considered to maximize the exogenous environmental influence by abolishing the endogenous component.

With respect to the human sample, the percentage of variance accounted for by the two-term function was not significantly different than that accounted for by the cosine function in any of the several conditions when one considers all the series. Nevertheless, in asymmetrical series of the free-running group, in which the endogenous component is maximized, the two-term function fit better than the cosine function. Aschoff (1992) pointed out that in free-running conditions, wakefulness as part of the sleep-wake cycle can be stretched and compressed within an extraordinary dilatability. It is possible that in such conditions the duration of the prevalence of thermogenic processes is also dilatable.

In relation to the basal conditions, human and animal samples presented a similar proportion of asymmetrical and symmetrical series. The present study cannot rule out the possibility that the better goodness-of-fit of the twoterm function was due to the experimental conditions; in the basal animal group, 12:12-h light:dark cycle was habitual, but control of light and darkness in the human basal group was either at the discretion of each subject or was based on the normal day-night routine of the subject.

Third, since the two-term function provides information of the timing involved in the processes of generation and loss of heat, it might be worthwhile to test it in an experimental setup in which these processes are manipulated. In the present study, constant routine and shiftwork human groups were the only groups for which these two processes could be affected, in the first case because of the deprivation of sleep and in the second because of the displacement of normal time of sleep. But in these cases, the difference between the percentage of variance accounted for by the two-term function and that accounted for by the cosine function did not reach statistical significance.

Fourth, this function can also model segments of a cycle, which constitutes another advantage since it is not necessary to have a complete cycle to utilize it. The function has been used to fit the oral temperature data, from 8:30 a.m. to midnight, that constitute the external validation of the morningness-eveningness questionnaire proposed by Horne and Östberg (1976). The two-term function discriminated between the timing of heat gain processes of 
neither-type subjects (i.e., those who have no morning and no evening event preference) and that of eveningtype subjects, and between the heat loss processes of evening-type subjects and those of morning-type subjects (Almirall \& Marcet, 1996).

Finally, although the two-term function is not a periodic one, it seems easy to complete it with a threshold mechanism that set on the thermogenic processes. Also, it would not be difficult to design an experimental setup in which 1 day favored thermal gain processes and another day favored thermal loss processes. Maybe in these conditions the circadian periodicity would be maintained, but the indexes $b / c$ would differ from day to day. This would be the testimony of the plasticity of the temperature regulation throughout the day.

In conclusion, the present study shows that the twoterm function is useful in modeling the body temperature throughout the day in those series whose shape is asymmetrical. Moreover, because it provides additional information to the cosine function, it is worthwhile to calculate and consider the two-term function as complementary to the cosine function.

\section{REFERENCES}

Almirall, H., \& Marcet, C. (1996). Individual differences in body temperature assessed by a two-term function. Perceptual \& Motor Skills, 83, 67-79.

Aschoff, J. (1992). On the dilatability of subjective time. Perspectives in Biology \& Medicine, 35, 276-280.

HORNE, J. A., \& ÖsTBERG, O. (1976). A self-assessment questionnaire to determine morningness-eveningness in human circadian rhythms. International Journal of Chronobiology, 4, 97-110.

Liv, L.-M., Hudak, G. B., Box, G. E. P., Muller, M. E., \& Tiao, G. C. (1985). The SCA statistical system. DeKalb, IL: Scientific Computing Association.

Minors, D. S., \& WATERHouse, J. M. (1988). Mathematical and statistical analysis of circadian thythms. Psychoneuroendocrinology, 1, 443-464.

Prodan, M. I. (1968). Forest biometrics. London: Academic Press.

REFINETTI, R. ( 1992). Analysis of the circadian rhythm of body temperature. Behavior Research Methods, Instruments, \& Computers, 24, 28-36.

WARREN, W. G. (1980). On removing the growth trend from dendrochronological data. Tree-Ring Bulletin, 40, 35-44.

(Manuscript received July 8, 1996; revision accepted for publication November 4, 1996.) 